\title{
Radical copolymerisation of chlorotrifluoroethylene with isobutyl vinyl ether initiated by the persistent perfluoro-3-ethyl-2,4-dimethyl-3-pentyl radical
}

\author{
G. J. Puts ${ }^{a}$, G. Lopez ${ }^{\mathrm{b}}$, T. Ono ${ }^{c}$, P. L. Crouse ${ }^{a}$ and B. M. Ameduri ${ }^{b^{*}}$
}

Results of the radical copolymerisation of chlorotrifluoroethylene (CTFE) with isobutyl vinyl ether (iBuVE) initiated by $\cdot \mathrm{CF}_{3}$ radicals generated by $\beta$-scission of perfluoro-3-ethyl-2,4-dimethyl-3-pentyl radical (PPFR) at $90{ }^{\circ} \mathrm{C}$ in a batch reactor are reported. ${ }^{19} \mathrm{~F}$ NMR spectroscopy enabled the assessment of the molecular weights of the poly(CTFE-alt-iBuVE) copolymer by end-group analysis. It was found that, at low initiator concentrations $\left(\leq 10 \mathrm{~mol} \%\right.$ ), the $\cdot \mathrm{CF}_{3}$ radicals preferably attack the vinyl ether monomer to initiate chain propagation and produce alternating poly(CTFE-alt-iBuVE) copolymers. At initiator ratios of $20 \mathrm{~mol} \%,{ }^{19} \mathrm{~F}$ NMR signals in the $\mathrm{CF}_{3}$ region other than the expected $\mathrm{CH}_{2}-\mathrm{CF}_{3}$ are observed and are attributed to $\cdot \mathrm{CF}_{3}$ addition patterns due to kinetic effects brought on by monomer solubility. The molecular weights for the copolymer produced from $1 \%, 5 \%$, and $10 \%$ PPFR were found to be 340000,237000 and $122000 \mathrm{~g} \cdot \mathrm{mol}^{-1}$, respectively. The copolymer produced from $20 \%$ PPFR was oligomeric in nature with a molecular weight of $18000 \mathrm{~g} \cdot \mathrm{mol}^{-1}$.

\section{Introduction}

Fluorinated polymers are niche macromolecules that play an integral role in modern life. ${ }^{1}$ They range from semi-crystalline to fully amorphous, and their uses span engineering thermoplastics and elastomers for the automotive and aeronautics industries, weather-proof coatings, biomedical materials, membranes for use in Li-batteries and fuel cells, and many more. ${ }^{2,3}$ Fluoropolymers possess exceptional physical and chemical properties, viz. high chemical-, thermal-, aging-, and weather resistance, as well as outstanding inertness to hydrocarbons, acids, and bases. Other highly desirable properties include low dielectric constant, low surface energy (water and oil repellency), low flammability, low refractive index, and low moisture absorption. Furthermore, the high strength of the $\mathrm{C}-\mathrm{F}$ bond $\left(485 \mathrm{~kJ} \cdot \mathrm{mol}^{-1}\right)$ grant fluoropolymers unparalleled resistance to oxidation. ${ }^{4-7}$

\footnotetext{
${ }^{a}$ Department of Chemical Engineering, University of Pretoria, Pretoria 0002, South Africa.

${ }^{b}$ Institut Charles Gerhardt, Ingénierie et Architectures

Macromoléculaires, UMR CNRS 5253, Ecole Nationale Supérieure de Chimie de Montpellier, 8 Rue de l'Ecole Normale, 34296 Montpellier, France. Correspondence to: Bruno Ameduri (E-mail:

bruno.ameduri@enscm.fr)

${ }^{c}$ National Institute of Advanced Industrial Science and Technology,

Research Institute of Instrumentation Frontier, 2266-98,

Anagahora, Shimoshidami, Moriyama, Nagoya, Aichi, 463-8560, Japan
}

Fluoropolymers also have their drawbacks. High crystallinity (up to $98 \%$ for PTFE) ${ }^{1,2,6}$ is often encountered in the homopolymers, resulting in poor solubility or total insolubility in common organic solvents and sparse solubility in fluorinated solvents. The poor solubility of fluorinated polymers and copolymers along with the general rigidity of their chains complicate the determination of molecular weight by the usual method of size exclusion chromatography (SEC).

The synthesis of fluorinated copolymers specially designed to overcome the problematic properties of the homopolymers has been the focus of extensive research. ${ }^{4,8}, 9$ Recently Patil et $a l .{ }^{10}$ utilised the persistent perfluoro-3-ethyl-2,4-dimethyl-3pentyl radical (abbreviated as PPFR $^{11}$, structure shown in Figure 1) to generate $\cdot \mathrm{CF}_{3}$ radicals for polymerisation initiation.

The resulting $\mathrm{CF}_{3}$ end groups were used in ${ }^{19} \mathrm{~F}$ NMR spectroscopy for the determination of average molecular weights of VDF copolymers. The technique provides $\mathbf{M}_{n}$ values closer to the true molecular weight of the copolymers than SEC. In addition $\mathrm{CF}_{3}$ labelling allows for the identification of the attack preferences of radical initiators on both comonomers.

In the research reported here, the use of PPFR as initiator was extended to the copolymers of CTFE and isobutyl vinyl ether. The molecular weights as well as the attack preferences of $\bullet \mathrm{CF}_{3}$ onto the co-monomers, and the polymer thermal properties have been studied. 


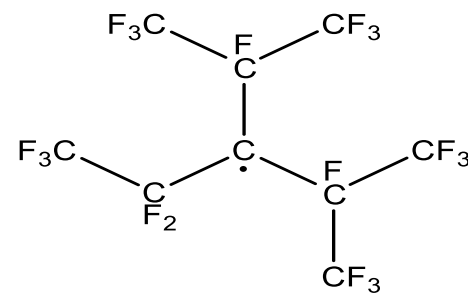

Figure 1 Structure of the perfluoro-3-ethyl-2,4-dimethyl-3pentyl persistent radical (PPFR).

\section{Experimental}

\section{Materials}

Chlorotrifluoroethylene $\geq 99 \%$ (CTFE, CAS No 79-38-9) was kindly provided by Honeywell (Buffalo, USA) and used as received. Isobutyl vinyl ether 99\% (iBuVE, CAS No 109-53-5), potassium carbonate $99.99 \%\left(\mathrm{~K}_{2} \mathrm{CO}_{3}, \mathrm{CAS}\right.$ No 584-08-7), dimethyl carbonate $99 \%$ (DMC, CAS No 616-38-6), methanol $\geq 99.8 \%\left(\mathrm{CH}_{3} \mathrm{OH}\right.$, ACS Reagent, Ph. Eur., CAS No 67-56-1) and acetone $\geq 99.5 \% \quad\left(\mathrm{CH}_{3} \mathrm{COCH}_{3}\right.$, ACS Reagent, Ph. Eur., CAS No 67-64-1) were purchased from Sigma-Aldrich (Saint Quentin-Fallavier, France) and used as received. Distilled water was provided by an in-house purification system.

The PPFR was kindly supplied by Prof. Taizo Ono of the Research Institute of Instrumentation Frontier in Nagoya. The PPFR was prepared by direct fluorination of a hexafluoropropene trimer precursor mixture at room temperature using undiluted fluorine gas. The PPFR solution was washed with $1 \mathrm{M}$ aqueous $\mathrm{Na}_{2} \mathrm{CO}_{3}$ followed by distilled water, and then distilled under reduced pressure $(25 \mathrm{mmHg})$. The distillate fraction boiling at $31-33{ }^{\circ} \mathrm{C}$ was used for this investigation. More information on the synthesis of the persistent radical may be found in Scherer et al. ${ }^{11}$

\section{Polymerisation apparatus}

The polymerisation reactions were conducted in a Parr Instruments (Moline, Illinois) stirred reactor (Hastalloy HC76). The reactor was equipped with an inlet valve and two outlet valves, a 3000 PSI rupture disc, a Span bourdon type pressure gauge and a thermowell. Temperature control was achieved by way of a heating jacket connected to a PID controller. A stainless steel sheathed (isolated) K-type thermocouple was used to monitor the temperature in the reactor.

\section{Radical polymerisation procedure}

The reactor was subjected to 24 hours of acetone wash at $90{ }^{\circ} \mathrm{C}$ before each polymerisation run and pressure tested at 20 bar nitrogen before being subjected to high vacuum for 1 hour.

The reaction mixture was prepared by dissolving $17.2 \mathrm{~g}$ of iBuVE and $0.0237 \mathrm{~g}$ of $\mathrm{K}_{2} \mathrm{CO}_{3}$ into $25 \mathrm{~mL}$ of DMC in a $50 \mathrm{~mL}$ round-bottom flask, with the PPFR initiator $(1 \%, 5 \%, 10 \%$ and $20 \%$, molar basis with respect to total monomer charge) being added after the potassium carbonate had dissolved. The reaction mixture was degassed under nitrogen for 20 minutes using the balloon and septum method. The reaction mixture was introduced to the reactor via a funnel tightly attached to the inlet valve with $25 \mathrm{~mL}$ of DMC used to wash out the degassing flask, ensuring that all the reagents were transferred to the vessel.

The reactor was immersed in liquid nitrogen until the DMC turned solid before the CTFE was transferred into the reactor. The mass of CTFE in the reactor was determined by weight difference (accurate to $0.5 \mathrm{~g}$ ). The mass of CTFE was kept constant at $20 \mathrm{~g}$ in all the experiments.

The loaded reactor was left in a fume hood to warm to 25 ${ }^{\circ} \mathrm{C}$ over a 1 hour period before it was placed in the reactor stand. The reaction temperature was increased in a stepwise fashion to avoid overheating by first heating to $40{ }^{\circ} \mathrm{C}$, then to $60{ }^{\circ} \mathrm{C}$, then $80{ }^{\circ} \mathrm{C}$ and finally to $90{ }^{\circ} \mathrm{C}$, stirring all the while. The reactor was left at $90{ }^{\circ} \mathrm{C}$ for 24 hours and afterwards cooled to $25{ }^{\circ} \mathrm{C}$ using an ice bath before it was degassed and opened.

In all cases, addition of PPFR to the solution of iBuVE in DMC resulted in an immediate yellowing of the solution. This is due to interaction between the $\pi$-orbitals of the vinyl ether and the PPFR radical ${ }^{12}$. This interaction was considered to have no effect on the polymerisation reaction.

\section{Product purification}

The product solution from the reactor was evaporated in a rotary evaporator, leaving behind a viscous material. The impure polymer material was thrice dissolved in acetone and evaporated, before being dissolved in sufficient acetone to produce a saturated solution which was precipitated by dropwise addition into a flask of vigorously stirred, cold $\left(\sim 0{ }^{\circ} \mathrm{C}\right)$ methanol. The precipitate was filtered off, washed with methanol, precipitated a second time, filtered off, washed with methanol and dried under high vacuum at $80{ }^{\circ} \mathrm{C}$. For the $20 \%$ PPFR experiment, distilled water rather than methanol was used as precipitant, as the product material dissolved in methanol.

The product from the 1\% PPFR experiment was isolated as a hard, whitish, opaque solid. The products from the $5 \%$ and $10 \%$ PPFR experiments were isolated as elastomeric, yellow to light orange solids and the product from the $20 \%$ PPFR experiment was isolated as a dark brown wax.

\section{NMR characterisation}

The nuclear magnetic resonance spectra were recorded on a Bruker AC 400 using deuterated chloroform. Coupling constants and chemical shifts are given in hertz $(\mathrm{Hz})$ and parts per million (ppm), respectively. ${ }^{1} \mathrm{H},{ }^{19} \mathrm{~F}$, and proton-decoupled ${ }^{19} \mathrm{~F}$ NMR were performed.

The experimental conditions for recording ${ }^{1} \mathrm{H}$, (or ${ }^{19} \mathrm{~F}$ ) spectra were: flip angle $90^{\circ}$ (or $30^{\circ}$ ); acquisition time of $4.5 \mathrm{~s}$ (or 0.7 s; , pulse delay of 2 s, 32 scans (or 1024 scans); and a pulse width of $5 \mu$ s for ${ }^{19} \mathrm{~F}$ NMR.

NMR samples were prepared by dissolving $100 \mathrm{mg}$ of polymer material in $1 \mathrm{~mL}$ of $\mathrm{CDCl}_{3}$. The ${ }^{19} \mathrm{~F}$ molecular weights 
were calculated using a similar procedure as previously reported $^{13}$.

\section{Thermogravimetric analysis (TGA)}

Thermogravimetric analyses under nitrogen were performed using a Perkin Elmer TGA 4000 coupled to a Perkin Elmer Spectrum 100 FTIR spectrometer. Polymer samples $(\sim 50 \mathrm{mg})$ were heated from $25{ }^{\circ} \mathrm{C}$ to $600{ }^{\circ} \mathrm{C}$ at $10{ }^{\circ} \mathrm{C} \cdot \mathrm{min}^{-1}$ in air flowing at a rate of $50 \mathrm{~mL} \cdot \mathrm{min}^{-1}$. The IR spectra were recorded from $550 \mathrm{~cm}^{-1}$ to $4000 \mathrm{~cm}^{-1}$ every 6 seconds at a resolution of $4 \mathrm{~cm}^{-1}$ Thermogravimetric analyses under air were carried out on a TGA 51 apparatus from TA Instruments. Polymer samples $(\sim 15$ $\mathrm{mg}$ ) were heated from $25{ }^{\circ} \mathrm{C}$ to $500{ }^{\circ} \mathrm{C}$ at $10{ }^{\circ} \mathrm{C} \cdot \mathrm{min}^{-1}$ in air flowing at a rate of $50 \mathrm{~mL} \cdot \mathrm{min}^{-1}$.

\section{Differential scanning calorimetry (DSC)}

Polymer samples $(\sim 10 \mathrm{mg})$ were subjected to two heat-cool cycles under a nitrogen flow of $50 \mathrm{~mL} \cdot \mathrm{min}^{-1}$. The polymer samples were heated from $25{ }^{\circ} \mathrm{C}$ to $150{ }^{\circ} \mathrm{C}$ at $10{ }^{\circ} \mathrm{C} \cdot \mathrm{min}^{-1}$, held isothermally at $150{ }^{\circ} \mathrm{C}$ for $5 \mathrm{~min}$, cooled from $150{ }^{\circ} \mathrm{C}$ to -150 ${ }^{\circ} \mathrm{C}$ at $10{ }^{\circ} \mathrm{C} \cdot \mathrm{min}^{-1}$, held isothermally at $-150{ }^{\circ} \mathrm{C}$ for $5 \mathrm{~min}$, subjected to another heat-cool cycle before being heated from $150{ }^{\circ} \mathrm{C}$ to $25^{\circ} \mathrm{C}$ at $10{ }^{\circ} \mathrm{C} \cdot \mathrm{min}^{-1}$. $\mathrm{T}_{\mathrm{g}}$ values were determined as the inflection point in the heat capacity jump.

\section{Size exclusion chromatography (SEC)}

Size exclusion chromatography was conducted using a GPC 50 from Polymer Labs (Now Agilent) with Cirrus software, as well as an Agilent 1260 Infinity system equipped with a Varian 390 LC triple detection system. The two systems used 2 PL Gel Mixed C columns $\left(200<\mathrm{Mw}<20 \mathrm{M} \mathrm{g} \cdot \mathrm{mol}^{-1}\right)$ with tetrahydrofuran (THF) as the eluent at a flow rate of 1.0 $\mathrm{mL} \cdot \mathrm{min}^{-1}$ at $35{ }^{\circ} \mathrm{C}$. The RI and UV detectors were calibrated using polystyrene standards. The viscometry detector used a universal calibration. Samples were prepared by dissolving 15 $\mathrm{mg}$ of polymer into $3 \mathrm{~mL}$ of THF followed by filtering through a $20 \mu \mathrm{m}$ commercial PTFE filter. Analyses were achieved by injection of $20 \mu \mathrm{L}$ filtered solution $\left(5 \mathrm{mg} \cdot \mathrm{mL}^{-1}\right)$.

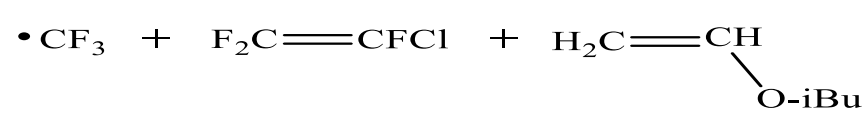

\section{Results and discussion}

The experimental conditions for the radical copolymerisation of chlorotrifluoroethylene (CTFE) with isobutyl vinyl ether (iBuVE) using PPFR as initiator, along with the characterisation results, are summarised in Table 1. PPFR has a half-life of 1 hour at $100{ }^{\circ} \mathrm{C}^{11}$, releasing ${ }^{\circ} \mathrm{CF}_{3}$ and a branched perfluorinated 2-pentene ( $\underline{\mathbf{A}})$ (Scheme 1).

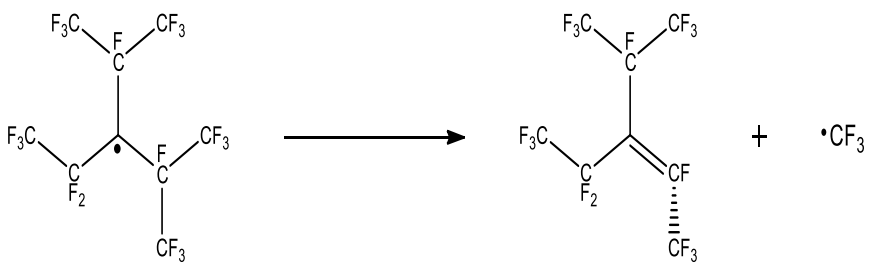

Scheme 1: $\beta$-scission elimination mechanism for the generation of $\bullet \mathrm{CF}_{3}$ from PPFR.

The $\mathrm{CF}_{3}$ radical initiates the radical copolymerisation of CTFE with iBuVE from $90^{\circ}$ (Scheme 2), taking into account that its half-life is 3 hours at this temperature. A $50 \mathrm{~mol} \%$ feed of CTFE/iBuVE was chosen since the maximum rate of polymerisation is found at this ratio ${ }^{14}$.

\section{NMR characterisation}

The progression of ${ }^{19} \mathrm{~F}$ NMR spectra going from $1 \%$ to $20 \%$ PPFR is presented in Figure 2 (full NMR spectra are available as supplementary material). The $\mathrm{CF}_{3}-\mathrm{CH}_{2}$ signal is observed at $-66 \mathrm{ppm}$ in the 1\% PPFR spectrum with no other signals observed in the $\mathrm{CF}_{3}$ range. Similarly for the 5\% and $10 \%$ PPFR spectra only the signal at $-66 \mathrm{ppm}$ is noted. For the $20 \%$ PPFR spectrum five signals are observed, viz. four major signals at $66,-77,-78$, and $-83 \mathrm{ppm}$. There is also one minor signal at -73 .

The ${ }^{1} \mathrm{H}$ NMR spectra for $1 \%$ and $10 \%$ PPFR are presented along with that of pure iBuVE in Figure 3. As expected from the literature ${ }^{15,16}$, both the ${ }^{19} \mathrm{~F}$ and ${ }^{1} \mathrm{H}$ NMR spectra displays signal broadening on the asymmetric carbons of CTFE and iBuVE units (in the range of -107 and -115 ppm for CTFE, and $4.5 \mathrm{ppm}$ for iBuVE).

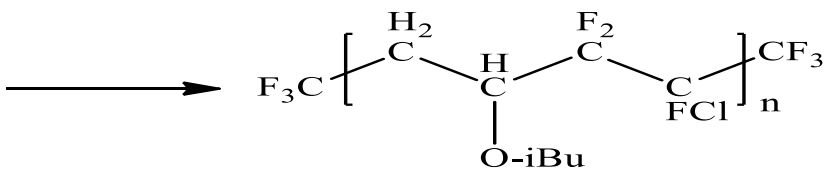

Scheme 2: Expected copolymerisation reaction of CTFE and iBuVE initiated by $\bullet \mathrm{CF}_{3}$ to yield a poly $(\mathrm{CTFE}-$ alt-iBuVE) alternating copolymer.

Table 1: Summary of experimental conditions and results obtained.

\begin{tabular}{|c|c|c|c|c|c|c|c|c|c|}
\hline Experiment & $\begin{array}{c}\text { Monomer } \\
\text { CTFE + iBuVE } \\
(\mathrm{mol})\end{array}$ & $\begin{array}{c}{[\text { PPFR }]_{0}} \\
{[C T F E]_{0}+[i B u V E]_{0}} \\
(\text { mol \%) }\end{array}$ & $\begin{array}{c}\text { Yield } \\
(\%)\end{array}$ & $\begin{array}{c}M_{n}{ }^{a} \\
(\mathrm{~g} / \mathrm{mol})\end{array}$ & $\begin{array}{c}\mathbf{M}_{\mathbf{n}}^{\mathbf{b}} \\
(\mathrm{g} / \mathrm{mol})\end{array}$ & DP & PDI & $\begin{array}{l}\mathbf{T}_{\mathrm{d}}^{\mathrm{c}} \\
10 \% \\
\left({ }^{\circ} \mathrm{C}\right) \\
\end{array}$ & $\begin{array}{c}\mathbf{T}_{\mathbf{g}}^{\mathbf{d}} \\
\left({ }^{\circ} \mathbf{C}\right)\end{array}$ \\
\hline 1 & $0.171+0.171$ & 1 & 64 & 85000 & 340000 & 1570 & 2.13 & 330 & 20 \\
\hline 2 & $0.171+0.171$ & 5 & 46 & 70000 & 237000 & 1100 & 1.58 & 340 & 23 \\
\hline 3 & $0.171+0.171$ & 10 & 58 & 66000 & 122000 & 560 & 1.62 & 344 & 24 \\
\hline 4 & $0.171+0.171$ & 20 & 20 & 59000 & 18000 & 84 & 2.19 & 322 & 23 \\
\hline
\end{tabular}

Number average molecular weight as determined by $\mathrm{SEC}^{\mathrm{a}}$ and ${ }^{19} \mathrm{~F} \mathrm{NMR}^{\mathrm{b}} ;{ }^{\mathrm{c}}$ decomposition temperature at $10 \%$ mass loss in air; ${ }^{\mathrm{d}}$ glass transition temperature. 


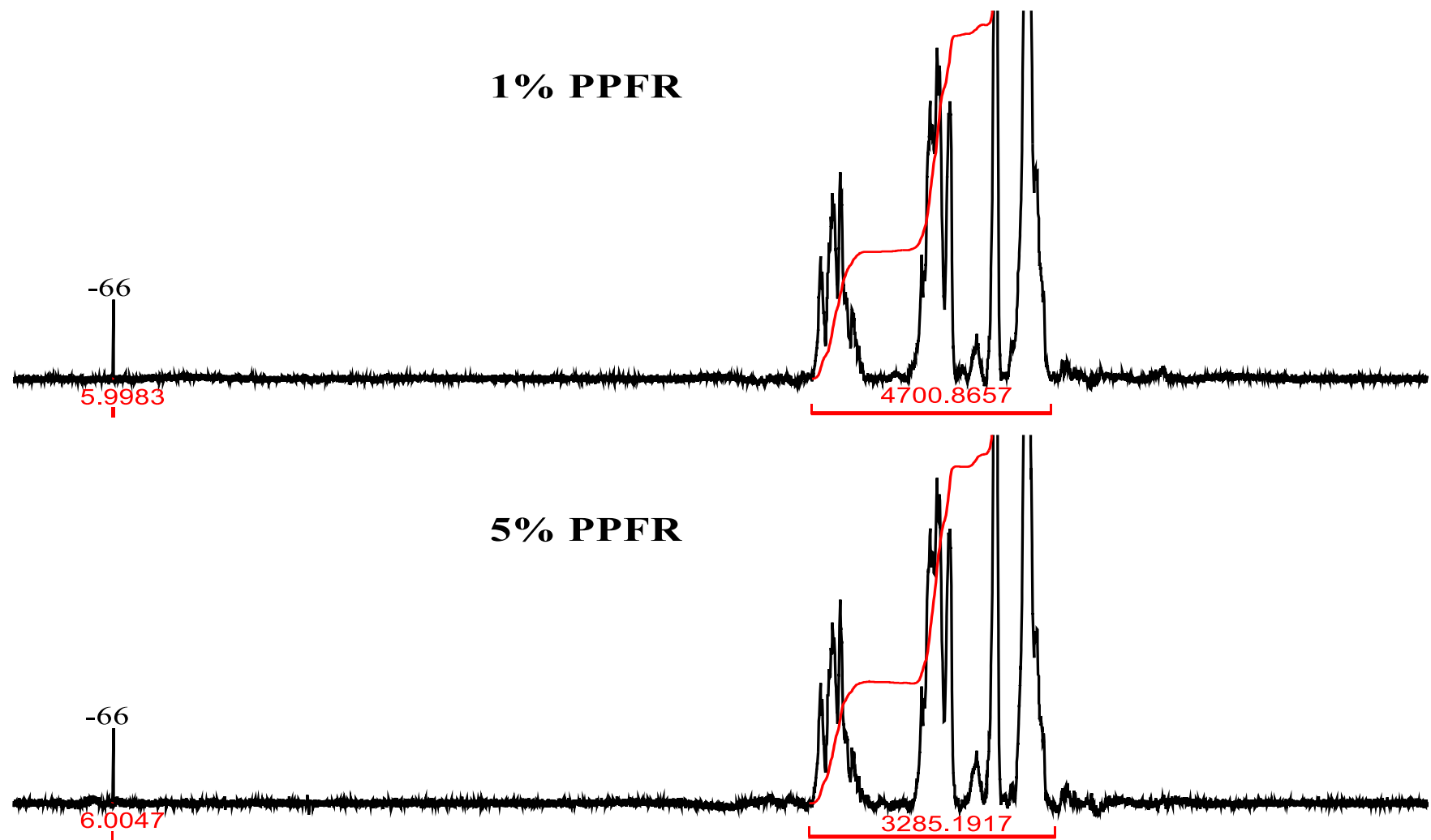

10\% PPFR

$20 \%$ PPFR
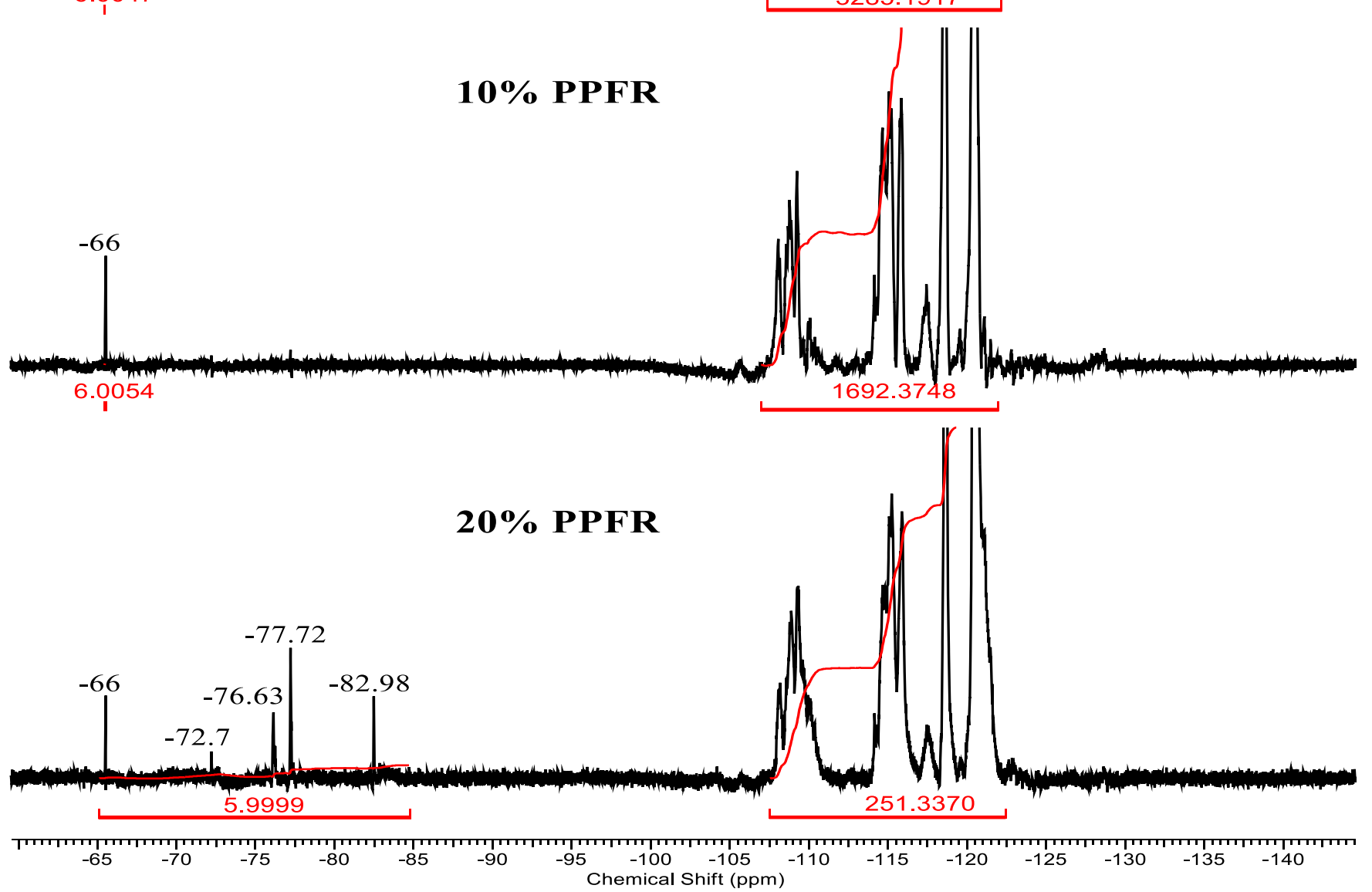

Figure 2: ${ }^{19} \mathrm{~F}$ NMR spectra of poly $\left(\mathrm{CTFE}-\right.$ alt-iBuVE) copolymers showing the progression of the $\mathrm{CF}_{3}{ }^{19} \mathrm{~F}$ NMR signal with increasing initiator concentration from $1 \mathrm{~mol} \%$ (top spectrum) to $20 \mathrm{~mol} \%$ (bottom spectrum). 


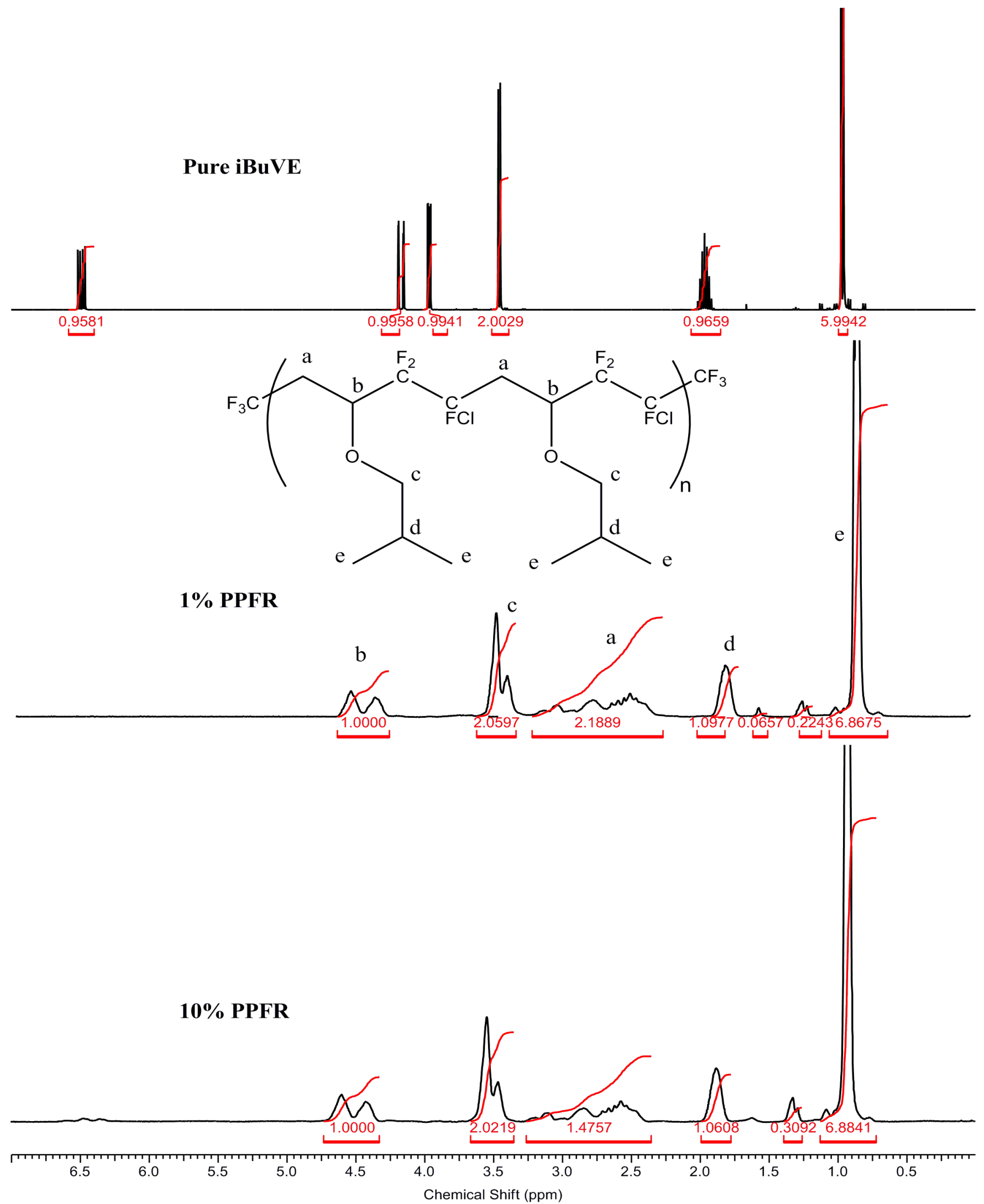

Figure 3: ${ }^{1} \mathrm{H}$ NMR spectra of poly(CTFE-alt-iBuVE) copolymers at $1 \%$ and $10 \%$ PPFR concentration compared to that of isobutyl vinyl ether (top spectrum). 
The spectra agree with what is expected for poly(CTFE-alt$\mathrm{iBuVE}$ ) copolymer and demonstrates that the system produced via $\bullet \mathrm{CF}_{3}$ radical initiation is alternating, as evidenced by the absence of any peaks in the $-127 \mathrm{ppm}$ range, indicative of $\mathrm{CFCl}$ groups in CTFE-CTFE dyads, in the ${ }^{19} \mathrm{~F}$ NMR spectrum ${ }^{16-19}$.

The five peaks present in the ${ }^{19} \mathrm{~F}$ NMR spectrum for $20 \%$ PPFR are unexpected. There are two plausible explanations: 1) The signals arise from $\bullet \mathrm{CF}_{3}$ additions to carbon sites other than $\mathrm{CH}_{2}$; or 2) the signals arise from CTFE or $\mathrm{BBuVE}$ reaction with the PPFR elimination products of transand cis-perfluoro-3-ethyl-4-methyl-2-pentene.

The ${ }^{1} \mathrm{H}$ NMR spectra (Figure 3) of poly(CTFE-alt-iBuVE) exhibit the absence of signals centered at 6.53, 4.20, and 3.95 ppm characteristic of unreacted VE vinylic protons (upper spectrum). However, their polymerised unit can be found between 2.50 and $3.2 \mathrm{ppm}$, and between 4.3 and $4.7 \mathrm{ppm}$ for the methylene and methyne protons, respectively $4,15,16,18,19$.

Both signals are broad arising from the presence of two types of asymmetric carbons leading to two diastereoisomers, which makes these protons anisochronous (i.e. nonequivalent). Methylene groups adjacent to the oxygen atoms, $\mathrm{CH}$ and both methyl groups in iBuVE are located at 3.45-3.65 ppm, 1.85 ppm, and 0.90 ppm, respectively.

There are several very small signals between 1.5 and $1 \mathrm{ppm}$. Several satellite signals near the signal assigned to the carbons are observed in the neat iBuVE spectrum. The small signals in the polymer spectra are ascribed to cumulative intensities of the small signals seen in the iBuVE spectrum. These signals are due to ${ }^{1} \mathrm{H}-{ }^{13} \mathrm{C}$ coupling.

\section{Addition preferences of $\bullet \mathrm{CF}_{3}$ radicals to the CTFE/iBuVE charge transfer complex}

The possible attack patterns of $\cdot \mathrm{CF}_{3}$ on the CTFE/iBuVE acceptor-donor complex ${ }^{5,17,20}$ are presented in Scheme 3. Of these possible addition pathways, path $1 \mathrm{a}$ is considered the most likely as the highly electrophilic $\bullet \mathrm{CF}_{3}$ radicals should preferentially attack the most electron donating, least hindered site in the monomer mixture ${ }^{10,21-23}$. In the case of the vinyl ether, known to be a donating monomer ${ }^{24}$, the preferred site will be the $\mathrm{CH}_{2}$ carbon, as it is not sterically hindered and is electron rich.

The literature ${ }^{10,} 13$ indicates that, if the polymerisation occurs in a regioselective way via path $1 \mathrm{a}$, there should be only one ${ }^{19} \mathrm{~F}$ NMR signal for $\mathrm{CF}_{3}$, centered at $-63 \mathrm{ppm}$.

Works done on the telomerisation of CTFE with $\mathrm{CF}_{3} \mathrm{CFClI}^{25,26}$ showed that the signals for $\mathrm{CF}_{3}$ should be in the region of $-77 \mathrm{ppm}$ if it forms part of the $\mathrm{CF}_{3} \mathrm{CFClCF}_{2}$ motif and should be in the region of $-82 \mathrm{ppm}$ if it forms part of the $\mathrm{CF}_{3} \mathrm{CF}_{2} \mathrm{CFCl}$ endgroup. If the other pathways (such as attack onto the CTFE) are sufficiently viable, then there should be ${ }^{19} \mathrm{~F}$ NMR signals at around -77 to $-78 \mathrm{ppm}$ as well. The assignments of the signals in the $20 \%$ PPFR spectrum and their relative abundance are presented in Table 2 .

The relative abundances were calculated by initially setting the integration of the $-66 \mathrm{ppm}$ signal to 6 and then dividing the integral of each $\mathrm{CF}_{3}$ signal by the sum of the integrations of all the $\mathrm{CF}_{3}$ signals.

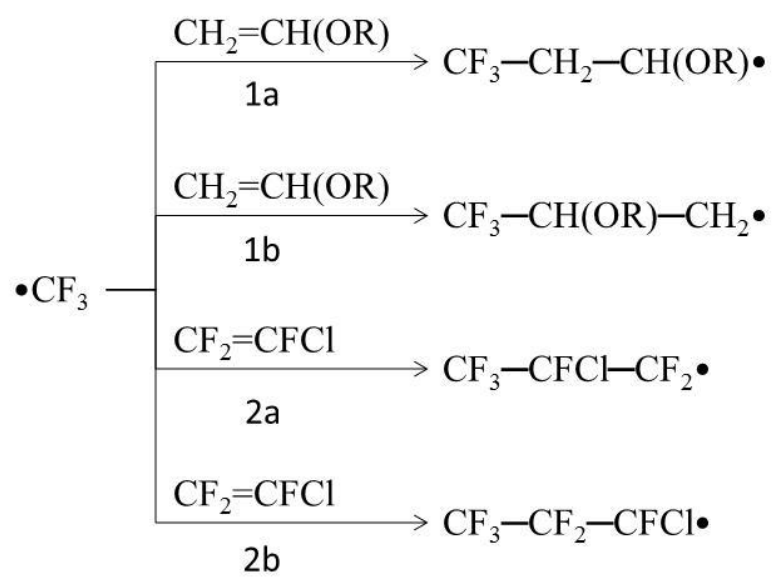

Scheme 3: Possible addition reactions of $\cdot \mathrm{CF}_{3}$ radicals onto the CTFE/iBuVE ${ }^{17,20}$.

Assuming that the signals in the ${ }^{19} \mathrm{~F}$ NMR spectrum for $20 \%$ PPFR are only due to different $\bullet \mathrm{CF}_{3}$ additions, then the NMR results show that at low initiator concentrations, pathway $1 \mathrm{a}$ is favoured, but at higher initiator concentrations pathway $2 \mathrm{a}$ becomes dominant. The low prevalence of attack via $2 \mathrm{~b}$ is due to electronic effects, the $\mathrm{CF}_{2}$ carbon being electron poor. The low occurrence of attack via $1 \mathrm{~b}$ is due to steric and electronic effects.

Table 2: $\mathrm{CF}_{3}$ signal assignments and percentage relative abundance in the copolymer made with $20 \%$ PPFR.

\begin{tabular}{ccc}
\hline $\begin{array}{c}\text { Chemical } \\
\text { Shift } \\
\text { (ppm) }\end{array}$ & Structural Assignment & $\begin{array}{c}\text { Relative } \\
\text { abundance } \\
(\boldsymbol{\%})\end{array}$ \\
\hline-66 & $\mathrm{CF}_{3}-\mathrm{CH}_{2}-\mathrm{CH}(\mathrm{O}-\mathrm{iBu})-\mathrm{CF}_{2}-\mathrm{CFCl}-\mathrm{R}$ & 6 \\
-73 & $\mathrm{C}_{3}-\mathrm{CH}(\mathrm{O}-\mathrm{iBu})-\mathrm{CH}_{2}-\mathrm{CF}_{2}-\mathrm{CFCl}-\mathrm{R}$ & 5 \\
-77 & $\mathrm{CF}_{3}-\mathrm{CFCl}-\mathrm{CF}_{2}-\mathrm{CH}(\mathrm{O}-\mathrm{iBu})-\mathrm{CH}_{2}-\mathrm{R}$ & 37 \\
-78 & $\underline{\mathrm{C}}_{3}-\mathrm{CFCl}-\mathrm{CF}_{2}-\mathrm{CH}_{2}-\mathrm{CH}(\mathrm{O}-\mathrm{iBu})-\mathrm{R}$ & 47 \\
-83.0 & $\mathrm{CF}_{3}-\mathrm{CF}_{2}-\mathrm{CFCl}-\mathrm{R}$ & 5 \\
\hline
\end{tabular}

${ }^{a}$ Assignments based on the NMR spectra for the telomerisation of $\mathrm{CF}_{2}=\mathrm{CFCl}$ with $\mathrm{CF}_{3} \mathrm{CFClI}$ and considerations from the literature ${ }^{25,27}$.

The effect of initiator concentration is remarkable in that there are no electronic or steric considerations that shift the regioselectivity away from $\mathrm{CH}_{2}$ towards $\mathrm{CFCl}$ attack. The previous work with VDF and PPFR was done in halogenated solvents, so the current reaction behaviour is possibly governed by kinetic effects arising from solubility considerations.

PPFR is a fully fluorinated species which dissolves in dimethylcarbonate (DMC) at low concentrations. However, at higher concentrations, a two phase system exists at standard conditions. The phase behaviour of large, sterically hindered, fully fluorinated molecules in contact with DMC at elevated temperatures and pressures is not known, but it is suspected that there exists, under the reaction conditions used here, a two 

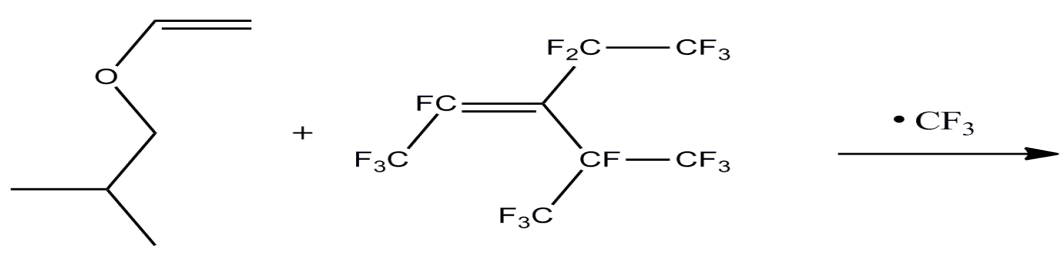

Scheme

4: $\quad$ Possible

$\underline{\underline{A}}$

phase system, one phase being DMC rich and the other being fluorous.

The solubility of CTFE in the fluorous phase should be an order of magnitude higher than its solubility in DMC. The "regioselectivity" at higher initiator concentrations is then ascribed simply to the much higher abundance of CTFE over iBuVE near to the initiator molecules.

\section{Reaction of iBuVE with PPFR elimination products}

At low initiator concentrations, any effect of the elimination products on the reaction is overshadowed by the much greater abundance of other monomers. However, at concentrations of $20 \%$ PPFR, the molar quantities of unsaturated fluorinated elimination product cannot be ignored when interpreting the results.

The decomposition reaction of PPFR is shown in Scheme 1. The by-product from $\bullet \mathrm{CF}_{3}$ elimination is a sterically hindered, unsaturated fluorocarbon. Normally, such hindered fluorocarbons do not homopolymerise under radical conditions, as is the case with hexafluoropropylene (HFP) or perfluoroalkylvinyl ethers (PAVEs). However, it has been shown that even HFP and PAVEs readily produces alternating copolymers with vinyl ethers ${ }^{28,29}$. Accordingly, there exists a distinct possibility that the iBuVE may react with the unsaturated elimination $\underline{\mathbf{A}}$ product to produce a copolymer. A possible reaction is shown in Scheme 4.

However, the terminal radical that would form on product $\underline{A}$ in such a reaction should be unreactive as its environment is sterically very similar to the radical centre of PPFR. A much more plausible scenario is the addition of iBuVE onto $\underline{A}$ to form a stable, radically capped monoadduct instead of a polymer.

If such a product did exist there should be ${ }^{19} \mathrm{~F}$ NMR signals at around $-175 \mathrm{ppm}$. Since no such signals are detected, this reaction did not occur and the unexpected ${ }^{19} \mathrm{~F}$ NMR signals for $\mathrm{CF}_{3}$ observed in the ${ }^{19} \mathrm{~F}$ NMR spectrum for $20 \%$ PPFR cannot be due to incorporation of the PPFR elimination product into the polymer.

\section{Effect of initiator concentration on molecular weight}

The normalised size exclusion chromatograms (SEC or GPC) are displayed in overlaid form in Figure 4. The expected decrease in molecular weight with increasing initiator ratio is noted. However, the concentration of initiator is observed to have limited effect on molecular weight beyond 5\%, displaying

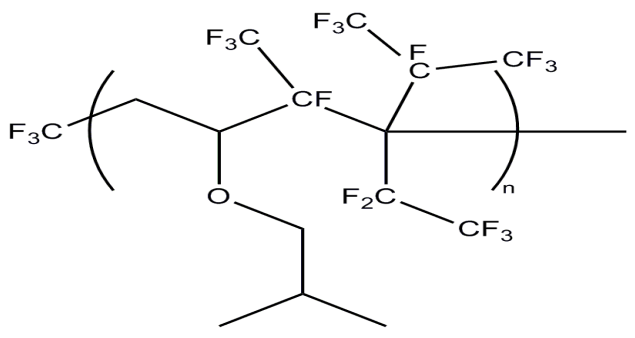

between iBuVE

and perfluoroolefin

$\underline{\mathbf{A}}$.

a significant drop in molecular weight from $1 \%$ to $5 \%$ (with $\mathrm{Mn}$ ranging from 85000 to $70000 \mathrm{~g} \cdot \mathrm{mol}^{-1}$ ), but showing a nearly linear correlation for decreasing molecular weight with increase of the square root of the ratio of initiator concentration to the monomer concentration from $5 \%$ to $20 \%$ (with Mn ranging from 66000 to $59000 \mathrm{~g} \cdot \mathrm{mol}^{-1}$ ). This trend is presented graphically in Figure 5.

The molecular weights determined by ${ }^{19} \mathrm{~F}$ NMR also show the expected decrease in molecular weight with increase in initiator concentration, with the trend being nearly linear in the region of $1 \%$ to $10 \%$ initiator (with Mn ranging from 340000 to $\left.122000 \mathrm{~g} \cdot \mathrm{mol}^{-1}\right)$.

As expected, since the GPC standards are polystyrene, there is a very large difference between the $M_{n}$ values derived from SEC (or GPC) and NMR.

\section{Thermal properties of $\mathrm{CF}_{3}$ terminated poly(CTFE-alt-iBuVE) copolymers}

5.1 Thermogravimetric analysis coupled to Fourier transform infrared spectroscopy. The thermograms for the thermal decomposition under nitrogen of the poly(CTFE-alt-iBuVE) alternating copolymers made with $1 \mathrm{~mol} \%, 5 \mathrm{~mol} \%$ and 10 mol\% PPFR are presented in Figure 6. FTIR spectra of the evolved gases from the thermal decomposition of the poly(CTFE-alt-iBuVE) alternating copolymers, taken at the point of maximum absorbance are presented in Figure 7.

The thermal degradation behaviour and degradation mechanism of fluoropolymers are dependent on the chemical nature of the polymer, the chain length and the morphology of the chains $^{30}$. For fully fluorinated polymers, the usual degradation routes involve either unzipping from the chain ends or breakdown due to random chain scission while for partially fluorinated polymers, dehydrofluorination is usually the main mechanism of degradation ${ }^{31,32}$. Polymers synthesised via nonfluorinated initiators are highly susceptible to unzipping from the chain ends or to oxidative attack initiated at the nonfluorinated chain ends.

The use of $\mathrm{CF}_{3}$ as terminal group enables the gauging of the degradation behaviour of poly(CTFE-alt-iBuVE) free from breakdown initiated at the chain ends.

The thermograms show that the polymer produced with 1 mol\% PPFR is the most stable, with a degradation onset temperature of $387{ }^{\circ} \mathrm{C}$, as is expected considering that its molecular weight is the largest of all the polymers. 
Interestingly, the $5 \mathrm{~mol} \%$ and $10 \mathrm{~mol} \%$ polymers show nearly the same degradation onset temperature $\left(367^{\circ} \mathrm{C}\right)$. The $20 \mathrm{~mol} \%$ polymer is not shown as the polymer underwent significant mass loss, via evaporation of the low molecular weight chains, long before the other polymers started degrading, with the onset of loss temperature for the higher molecular weight fraction of the $20 \mathrm{~mol} \%$ PPFR polymer being in the region of $325^{\circ} \mathrm{C}$.

The $10 \%$ mass loss temperature under air is reported in Table 1, and demonstrates that the thermal behaviour of the 5 mol\% and 10 mol\% polymers are nearly identical under both air and nitrogen. The differences in onset temperatures between heating in air and in inert atmosphere demonstrates that the alternating poly(CTFE-alt-iBuVE) copolymer undergoes combustion long before the polymer chains start degrading, implying that in the event of combustion during service, the subsurface polymer material will remain in serviceable condition when the surface combustion is extinguished. Also, the differences in degradation onset temperatures between the 1 mol\% PPFR, $5 \mathrm{~mol} \%$ and $10 \mathrm{~mol} \%$ PPFR polymers demonstrates that the degradation behaviour is dependent on molecular weight.

Furthermore, these results indicate that the intrinsic oxidative thermal stability of the poly(CTFE-alt-iBVE) backbone is quite good for a partially fluorinated polymer, being comparable to the stability achieved by fully fluorinated polymers. For comparison, the onset of degradation temperature for PTFE is around $550{ }^{\circ} \mathrm{C}^{33}$ under the same conditions.

The infrared spectra of the evolved gases display the expected release of $\mathrm{HF}$ and $\mathrm{HCl}$. The infrared absorbance bands for $\mathrm{CO}$ and $\mathrm{CO}_{2}$ are also observed, at 2142 and $2336 \mathrm{~cm}^{-1}$, respectively. The peak at 1748, 3084, 2964 and $1116 \mathrm{~cm}^{-1}$ is indicative of a $\mathrm{C}=\mathrm{O}$ group, a $\mathrm{C}=\mathrm{C}-\mathrm{H}$ stretch, a $\mathrm{CH}_{2}$ stretch and of $=\mathrm{C}-\mathrm{H}$ bends, respectively. Also, no peaks for an $\mathrm{O}-\mathrm{H}$ bond, usually seen at around $3600 \mathrm{~cm}^{-1}$ are observed.

While the gas phase is obviously a mixture of species, the FTIR spectra indicate that the major component of the organic pyrolysis products is something akin to isobutenal. Given that $\mathrm{CO}$ and $\mathrm{CO}_{2}$ are also produced, other minor components like isobutylene and ethers of isobutene must also be present in the gas phase. The literature ${ }^{31,32}$ indicates that monofluorides and monochlorides of these compounds should also be present in some small quantity.

The work of Zulfiqar et al. ${ }^{31,32,34}$ focused on the random copolymers of CTFE with methyl methacrylate, VDF and styrene. These polymers are all random copolymers. It was noticed there that the degradation of CTFE-MMA copolymers occurs in a two-step process, with $\mathrm{HCl}$ being eliminated first, followed by depolymerisation of the polymer chain to MMA and chlorofluorocarbons. No mention is made of the elimination of HF.

The thermal decomposition of polyvinyl alcohol and polyvinyl acetate (model polymers approximating vinyl ethers ) also occur in a two-step process. Polyvinyl alcohol first releases water, forming double bonds in the polymer chain and afterward undergoing chain scission to produce unsaturated aldehydes and ketones. Poly vinyl acetate first releases acetic acid to form an unsaturated, ketenic polymer backbone, followed by elimination of ketones from the unsaturared chain $^{35,36}$.

In the case of poly(CTFE-alt-iBuVE), the breakdown is seen to be one step with $\mathrm{HCl}, \mathrm{HF}$ and the pendant ether group eliminated from the polymer chain concurrently, with minimal internal rearrangements and reactions of the pendant ether groups with the radicals in the polymer backbone.

5.2 Differential scanning calorimetry (DSC). The main calorimetric parameter of importance to poly(CTFE-alt-iBVE) copolymers is the glass transition temperature, which in this case is shown to be nearly independent of polymer molecular weight. The glass transition temperatures for poly(CTFE-altiBVE) copolymers are around $23{ }^{\circ} \mathrm{C}$. This invariance is in agreement with the expected behaviour ${ }^{37}$, given as that the glass transition is not so much dependent on molecular weight as it is dependent on polymer composition, which, in this case remains constant at a 1:1 ratio of CTFE:iBuVE.

\section{Conclusions}

A series of poly(CTFE-alt-iBuVE) alternating copolymers has been synthesised in good yield via radical polymerisation initiated by $\cdot \mathrm{CF}_{3}$ released from the $\beta$-scission of perfluoro-3ethyl-2,4-dimethyl-3-pentyl persistant radical at $90{ }^{\circ} \mathrm{C}$. The addition behaviour of $\cdot \mathrm{CF}_{3}$ radicals onto the CTFE/iBuVE is highly dependent on the initiator-to-monomer ratio. It was demonstrated that $\cdot \mathrm{CF}_{3}$ radicals preferentially attack the methylene site in the vinyl ether monomer to initiate chain cross propagation when using low initiator concentrations. At initiator concentrations of $20 \%$ it was shown that there is significant deviation from the expected behaviour, ascribed to either the effects of initiator. The usefulness of $\mathrm{CF}_{3}$ end groups as labels for molecular weight determination in poly(CTFE-altiBVE) copolymers by ${ }^{19} \mathrm{~F}$ NMR was demonstrated and compared to results obtained by SEC. The polymerisation initiated by PPFR persistent radical exhibiting the expected tendency for the molecular weight of the copolymers to increase with decreasing initiator concentration.

\section{Acknowledgements}

The authors thank the National Research Foundation of South Africa, CNRS, France and the south African Department of Science and Technology's Fluorochemical Expansion Initiative for the opportunity to collaborate and for funding this research, and Honeywell company for supplying the CTFE. 


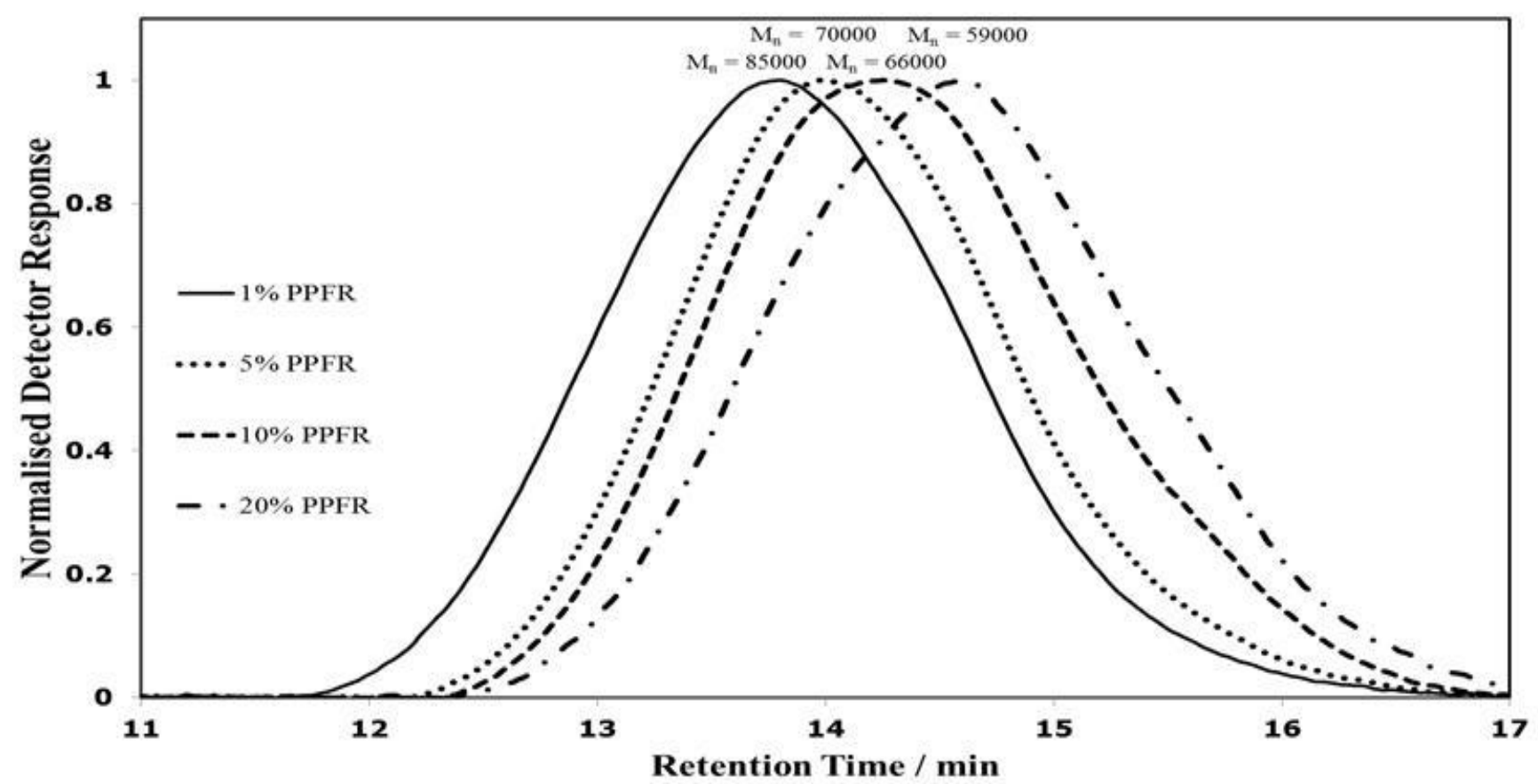

Figure 4: Size exclusion chromatograms showing the number average molecular weights of poly(CTFE-alt-iBuVE) copolymers prepared from various amounts of PPFR radical molar percentages.

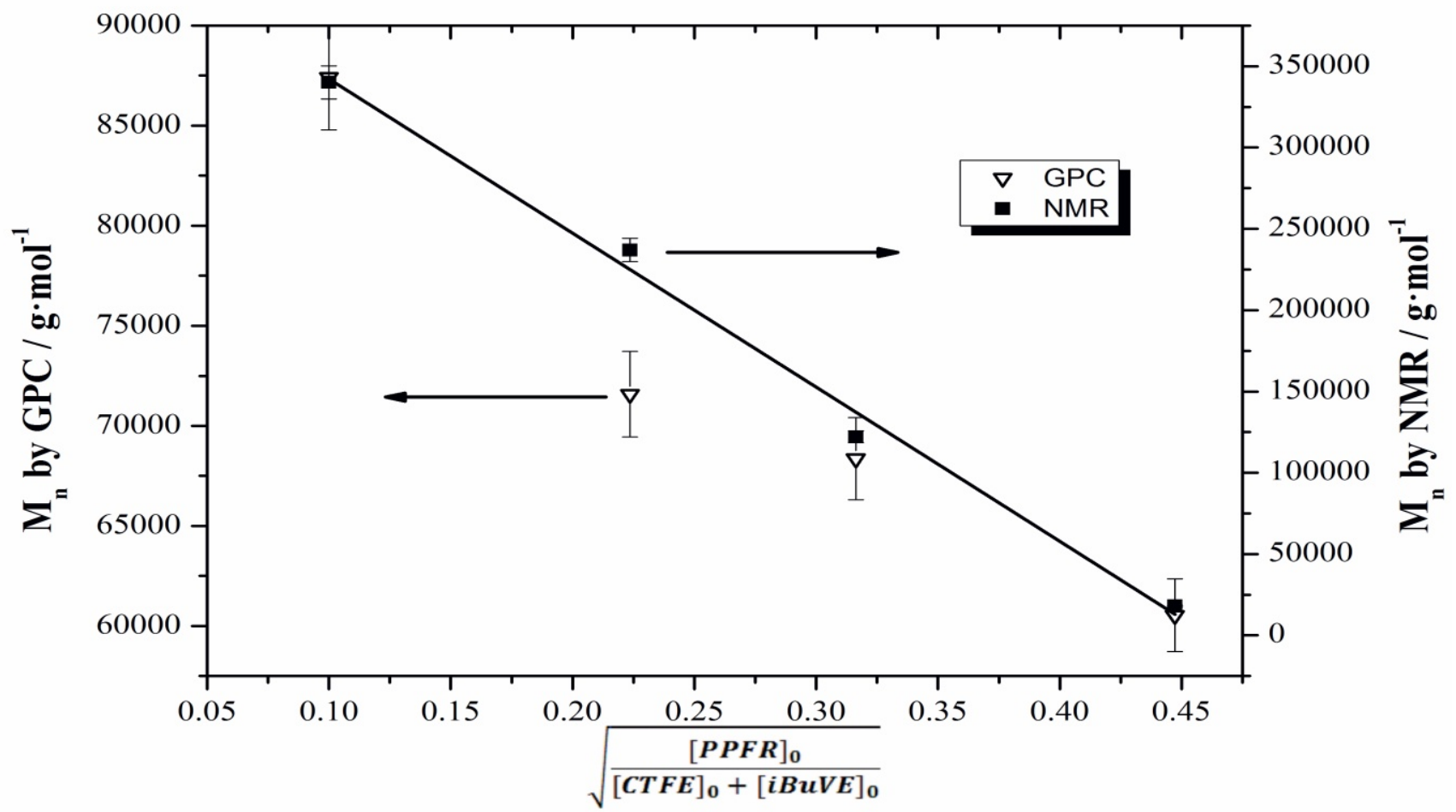

Figure 5: Correlation of $M_{n}$ decrease as PPFR ratio increases as determined by both SEC $(\Delta)$ and ${ }^{19}$ F NMR spectroscopy ( $\left.\boldsymbol{\square}\right)$. 


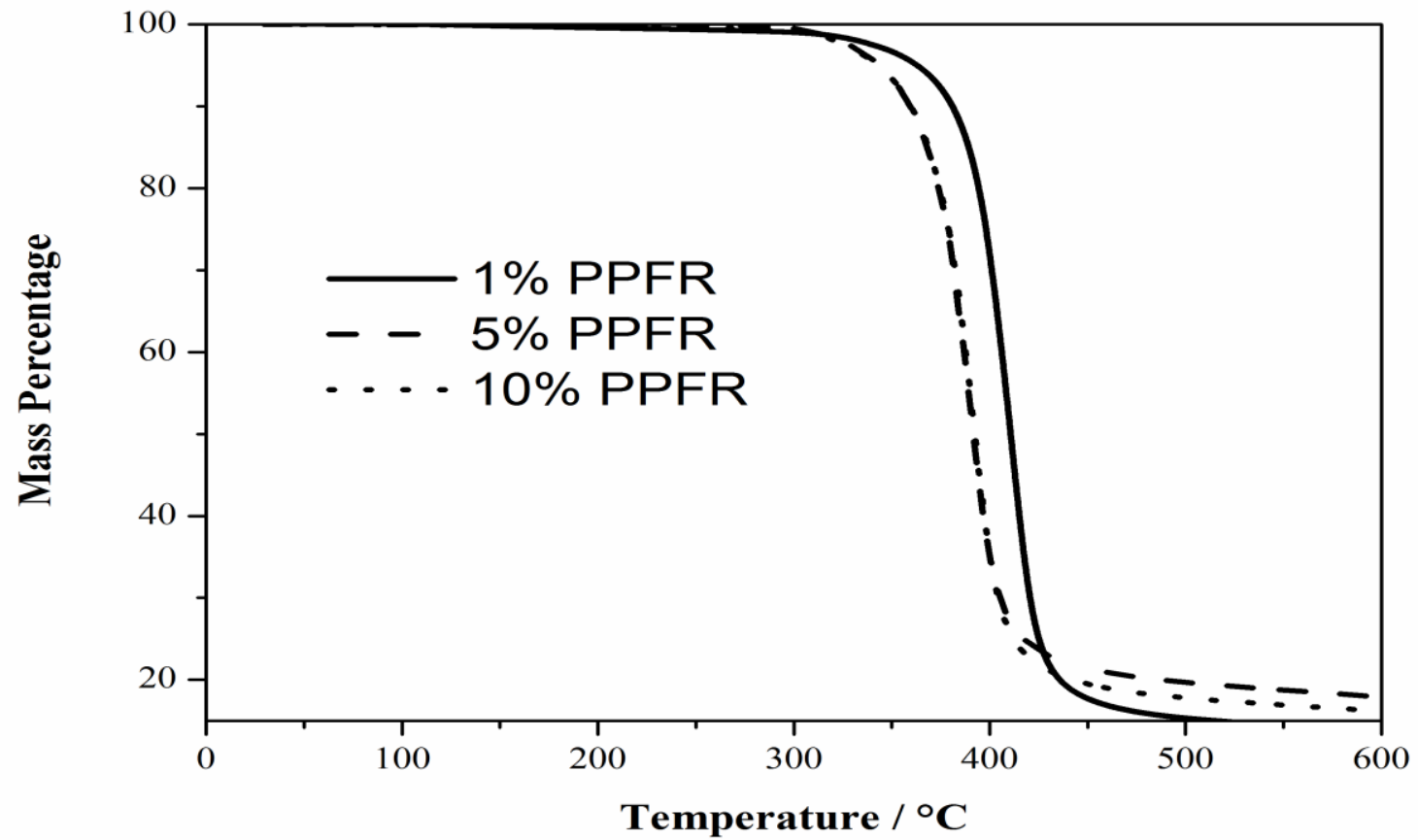

Figure 6: TGA thermograms for the poly(CTFE-alt-iBVE) copolymers under $\mathrm{N}_{2}$ at $10{ }^{\circ} \mathrm{C} / \mathrm{min}$.

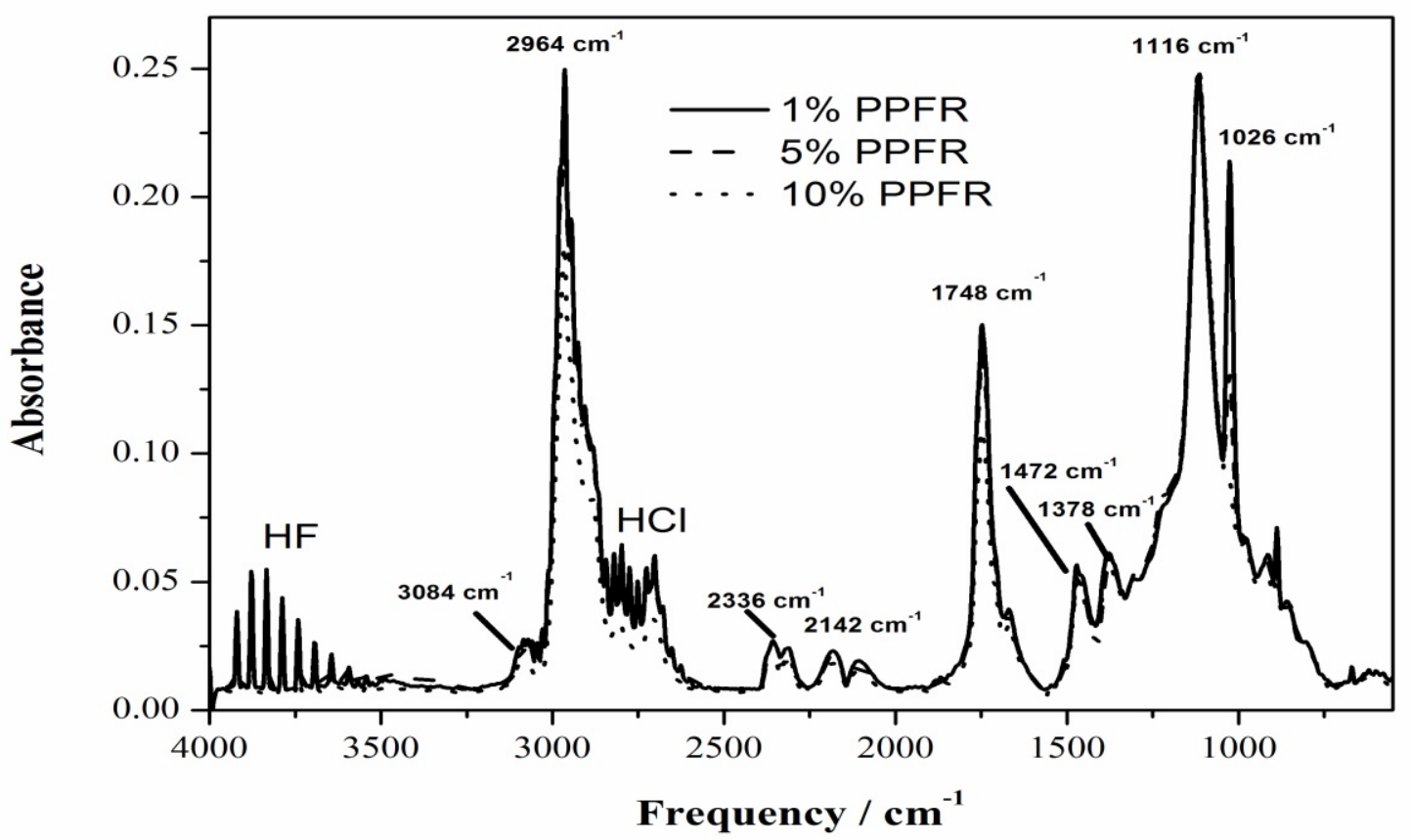

Figure 7: FTIR spectrum of the evolved gases from the thermal decomposition of the poly(CTFE-alt-iBuVE) alternating copolymers, taken at the point of maximum absorbance, showing, among other species, the evolution of $\mathrm{HF}, \mathrm{HCl}, \mathrm{CO}$ and $\mathrm{CO}{ }_{2}$. 


\section{Notes and references}

1. J. G. Drobny, Technology of Fluoropolymers, CRC Press, Boca Raton, 2014.

2. S. Ebnesajjad, Fluoroplastics, Volume 1: Non-Melt Processible Fluoroplastics, Elsevier Science, Amsterdam, 2000

3. S. Ebnesajjad, Fluoroplastics, Volume 2: Melt Processible Fluoroplastics: The Definitive User's Guide, Elsevier Science, Amsterdam, 2002.

4. F. Boschet and B. Ameduri, Chem. Rev., 2013, 114, 927-980.

5. B. Ameduri and B. Boutevin, Well-Architectured Fluoropolymers: Synthesis, Properties and Applications: Synthesis, Properties and Applications, Elsevier Science, 2004.

6. G. Hougham, P. E. Cassidy, K. Johns and T. Davidson, Fluoropolymers 1, Springer US, New York, 1999.

7. D. W. Smith, S. T. Iacono and S. S. Iyer, Handbook of Fluoropolymer Science and Technology, Wiley, New York, 2014.

8. B. Ameduri, Chem. Rev., 2009, 109, 6632-6686.

9. Y. Patil, T. Ono and B. Ameduri, ACS Macro Lett., 2012, 1, 315 320.

10. Y. Patil, A. Alaaeddine, T. Ono and B. Ameduri, Macromolecules, 2013, 46, 3092-3106.

11. K. V. Scherer, T. Ono, K. Yamanouchi, R. Fernandez and P. Henderson, J. Am. Chem. Soc., 1985, 107, 718-719.

12. T. Ono and K. Ohta, J. Fluorine Chem., 2014, 167, 198-202.

13. F. Boschet, T. Ono and B. Ameduri, Macromol. Rapid Commun., 2012, 33, 302-308.

14. Y. Tabata and T. A. Du Plessis, Journal of Polymer Science Part A-1: Polymer Chemistry, 1971, 9, 3425-3435.

15. G. Couture, B. Campagne, A. Alaaeddine and B. Ameduri, Polym Chem., 2013, 4, 1960-1968.

16. A. Alaaeddine, G. Couture and B. Ameduri, Polym. Chem., 2013, 4, 4335-4347.

17. G. V. D. Tiers and F. A. Bovey, J. Polym. Sci., Part A: General Papers, 1963, 1, 833-841.

18. M. Gaboyard, Y. Hervaud and B. Boutevin, Polym. Int., 2002, 51, 577-584.

19. D. Carnevale, P. Wormald, B. Ameduri, R. Tayouo and S. E. Ashbrook, Macromolecules, 2009, 42, 5652-5659.

$20 . \quad$ Z. M. O. Rzaev, Prog. Polym. Sci., 2000, 25, 163-217.

21. Y. Takeyama, Y. Ichinose, K. Oshima and K. Utimoto, Tetrahedron Lett., 1989, 30, 3159-3162.

$22 . \quad$ J. N. Cape, A. C. Greig, J. M. Tedder and J. C. Walton, J. Chem. Soc. Faraday Trans. 1: Phys. Chem. Cond. Phases, 1975, 71, $592-$ 601.

23. K. Miura, Y. Takeyama, K. Oshima and K. Utimoto, Bull. Chem. Soc. Jpn., 1991, 64, 1542-1553.

24. D. J. T. Hill, J. J. O'Donnell and P. W. O'Sullivan, Prog. Polym. Sci., 1982, 8, 215-275.

25. M. P. Amiry, R. D. Chambers, M. P. Greenhall, B. Ameduri, B. Boutevin, G. Caporiccio, G. A. Gornowicz and A. P. Wright, The peroxide initiated telomerization of Chlorotrifluoroethylene with perfluorochloroalkyl iodides, Polym. Prep. (ACS Polym. Div.), 1993, 34, 411-412.

26. R. N. Haszeldine, J. Chem. Soc., 1955, 4291-4302.

27. W. R. Dolbier, Guide to Fluorine NMR for Organic Chemists, Wiley, New York, 2009.

28. D. Valade, F. Boschet and B. Améduri, Macromolecules, 2009, 42, 7689-7700.

29. A. Alaaeddine, F. Boschet, B. Ameduri and B. Boutevin, $J$. Polym. Sci., Part A: Polym. Chem., 2012, 50,3303-3312.

30. T. R. Crompton, Thermo-oxidative Degradation of Polymers, iSmithers, Akron, Ohio, 2010.

31. S. Zulfiqar, M. Rizvi, A. Munir, A. Ghaffar and I. C. McNeill, Polym. Degrad. Stab., 1996, 52, 341-348.

32. S. Zulfiqar, M. Zulfiqar, M. Rizvi, A. Munir and I. C. McNeill, Polym. Degrad. Stab., 1994, 43, 423-430.

33. G. J. Puts and P. L. Crouse, J. Fluorine Chem., 2014, 168, 260267.

34. S. Zulfiqar, M. Rizvi and A. Munir, Polym. Degrad. Stab., 1994, 44, 21-25.

35. B. J. Holland and J. N. Hay, Polymer, 2002, 43, 2207-2211.
36. B. J. Holland and J. N. Hay, Polymer, 2001, 42, 6775-6783.

37. J. W. Nicholson, The Chemistry of Polymers, Royal Society of Chemistry, London, 2012. 


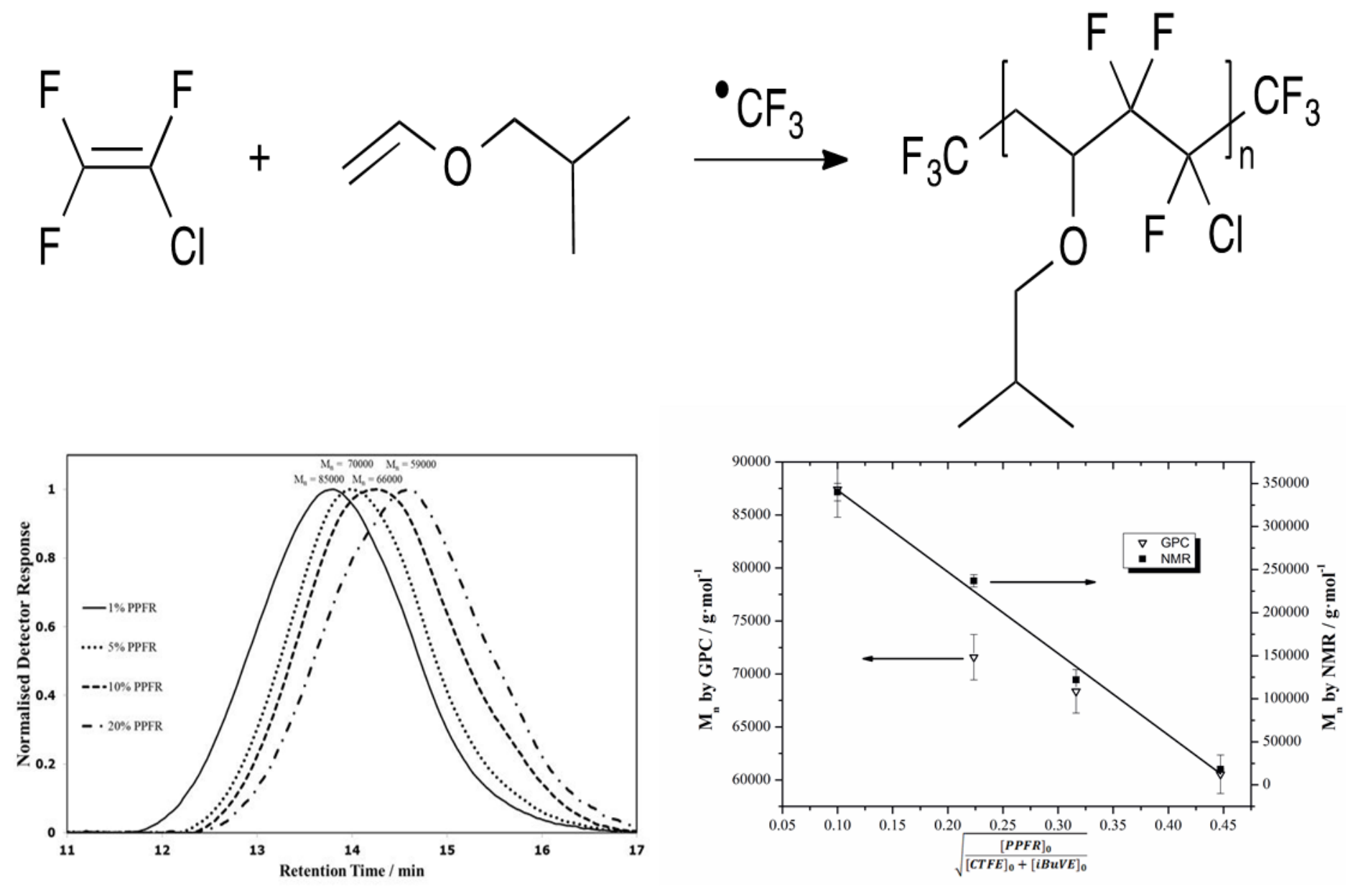

